\title{
Correlation Between Lacunae and the Wearing-off Phenomenon in Parkinson's Disease
}

\author{
Meimei Zhang ${ }^{1,2}$ \\ Huimin Chen ${ }^{3}$ \\ Genliang Liu ${ }^{1,2}$ \\ Xuemei Wang ${ }^{1,2}$ \\ Zhan Wang ${ }^{1,2}$ \\ Tao Feng ${ }^{1,2}$ \\ Yumei Zhang ${ }^{2,4}$
}

'Department of Neurology, Beijing Tiantan Hospital, Capital Medical University, Beijing, People's Republic of China; ${ }^{2}$ China National Clinical Research Center for Neurological Diseases (NCRC-ND), Beijing, People's Republic of China; ${ }^{3}$ Department of Neurology, Beijing Hospital, National Center of Gerontology, Beijing, People's Republic of China; ${ }^{4}$ Department of Rehabilitation, Beijing Tiantan Hospital, Capital Medical University, Beijing, People's Republic of China
Correspondence: Tao Feng Department of Neurology, Beijing Tiantan Hospital, Capital Medical University, No. 119 South 4th Ring West Road, Fengtai District, Beijing, 100070, People's Republic of China

Email bxbkyjs@sina.com

Yumei Zhang

Department of Rehabilitation, Beijing

Tiantan Hospital, Capital Medical

University, No. II 9 South 4th Ring West

Road, Fengtai District, Beijing, 100070 ,

People's Republic of China

Email zhangyumei95@aliyun.com
Purpose: Lacunae are imaging biomarkers of cerebral small vessel disease (CSVD) and are correlated with the degree of gait instability in Parkinson's disease (PD). The wearing-off phenomenon (WO) occurs more frequently in PD patients as disease progresses. The present study aimed to investigate the overall impact of the quantity and location of lacunae on the WO in PD.

Patients and Methods: This retrospective, single-center study included 315 consecutive eligible patients with PD from Beijing Tiantan Hospital from May 2016 to August 2018. We collected data on demographics and clinical features, assessed lacunae and examined the presence of the WO. The association between lacunae and the WO was assessed using a binary logistic regression model.

Results: The number of lacunae was significantly associated with the WO in patients with PD according to a model adjusted for age at onset, disease duration, Hoehn-Yahr (H-Y) staging, Movement Disorder Society-Unified Parkinson's Disease Rating Scale part III (MDS-UPDRS III) total score and levodopa equivalent daily dosage (LEED) ( $\mathrm{P}=0.037$, OR $1.156,95 \% \mathrm{CI}$ $1.009,1.325)$ and to a model further adjusted for other CSVD imaging biomarkers $(\mathrm{P}=0.046, \mathrm{OR}$ $1.172,95 \%$ CI 1.003, 1.369). Following additional adjustment for other potential confounders, the association remained significant $(\mathrm{P}=0.043$, OR $1.195,95 \%$ CI $1.005,1.421)$. Lacunae in subcortical areas $(\mathrm{P}=0.004$, OR $0.498,95 \%$ CI $0.308,0.803)$ and basal ganglia $(\mathrm{P}=0.046, \mathrm{OR}$ $1.616,95 \%$ CI $1.009,2.587)$, especially in the caudate nuclei ( $\mathrm{P}=0.023$, OR $1.104,95 \%$ CI 0.185 , 0.881 ), were significantly associated with the WO in PD patients.

Conclusion: Our finding highlights the significant association between lacune and the WO, and lacunae may be an independent contributor to the WO in PD patients. Promoting neurovascular health may prevent the progression of the WO in PD patients.

Keywords: Parkinson's disease, wearing-off phenomenon, lacunae, cerebral small vessel disease

\section{Introduction}

Resting tremor, bradykinesia, rigidity and gait/postural instability are the main symptoms of Parkinson's disease (PD), and levodopa replacement therapy is the primary method for alleviating these symptoms. ${ }^{1}$ As the disease progresses, these clinical symptoms worsen and are accompanied by the gradual development of drug-related motor complications. The wearing-off phenomenon (WO) is a motor fluctuation that occurs within several years in most PD patients who have undergone chronic dopamine replacement therapy. ${ }^{2}$ The timing of the development of the WO varies among patients as the disease progresses. According to the literature, approximately $20-40 \%$ of PD patients will experience the WO after 4-6 years of dopamine replacement therapy. ${ }^{3}$ 
According to previous studies, the risk factors for WO mainly included female sex, age at onset of PD, disease duration, dose of daily levodopa, and Unified Parkinson's Disease Scale Part II and Part III scores. ${ }^{4,5}$ However, these factors are limited in terms of the demographic and clinical features of PD patients.

The correlation between cerebral small vessel disease (CSVD) and PD has received increasing attention in recent years. Several studies have shown that CSVD may be a comorbidity of PD and could worsen the motor symptoms and cognition of PD patients, ${ }^{6-9}$ although the underlying mechanism remains largely unknown. A widely accepted hypothesis is that the CSVD lesion disrupt the striato-thalamo-cortical circuit, ${ }^{10}$ which includes the subthalamic nucleus, pedunculopontine nucleus, and visuospatial and executive networks and is thought to be involved in the pathophysiology of gait and postural impairment in patients with PD. ${ }^{11}$ Vascular dysfunction accelerates the processes of neuronal dysfunction or death and neurodegeneration may engage in the mechanism, too. ${ }^{12}$ However, vascular pathology was shown to be significantly lower in autopsy-proven PD than in normally aging persons, ${ }^{13}$ and some vascular risk factors, such as smoking, may even play a protective role in PD in terms of development or prognosis. ${ }^{14}$

Additionally, cerebrovascular disease has been identified at autopsy as an etiological factor in parkinsonism in $1-3.2 \%$ of cases, and white matter hyperintensity (WMH), an imaging biomarker of CSVD, could affect gait and cognitive function in patients with PD based on MRI evidence. $^{15,16}$

Lacunae are another imaging biomarkers of CSVD. ${ }^{10}$ Our previous study indicated that lacunae were correlated with gait instability in PD. ${ }^{17}$ We hypothesize that lacunae may also contribute to the $\mathrm{WO}$, but to the best of our knowledge, no previous study has explored the association between lacunae and the WO in PD.

Therefore, the aim of this study was to estimate this association in PD patients and identify a new strategy for resolving the $\mathrm{WO}$ in $\mathrm{PD}$.

\section{Patients and Methods}

\section{Patients}

This was a retrospective, single-center study from the Department of Neurology of Beijing Tiantan Hospital, Capital Medical University. From May 2016 to August 2018, a total of 315 idiopathic PD patients who underwent MRI scans were enrolled in this study. Patients who met the clinically definite PD based on the 2015 Movement Disorder Society (MDS) Clinical Diagnosis Criteria were included. ${ }^{18} \mathrm{~A}$ reported good response to levodopa or $>30 \%$ improvement in the response to levodopa was identified to differentiate PD from vascular parkinsonism. The exclusion criteria included 1) uncertain PD diagnosis or suspicion of atypical parkinsonism (multiple system atrophy, corticobasal ganglionic degeneration, or progressive supranuclear palsy) and second parkinsonism syndrome (vascular, drug-induced, toxin-induced, postinfectious parkinsonism); 2) a history of moderate-tosevere head trauma, hydrocephalus, brain surgery, or brain tumor; and 3) a lack of clinical and MRI data. This study was approved by the Ethics Committee of the Beijing Tiantan Hospital and was performed in accordance with the Declaration of Helsinki. Informed consent was obtained either from the participants or their closest relatives.

\section{Clinical Assessment}

Demographic information (age at admission, sex, weight and height), medical history (hypertension, diabetes, cardiac disease [angina, myocardial infarction, or heart failure), smoking history, drinking history and disease duration were collected. The results of admission blood tests for factors associated with cerebrovascular disease (triglyceride [TG], total cholesterol [TC], low-density lipoprotein [LDL], high-density lipoprotein [HDL], homocysteine $[\mathrm{HCY}]$, uric acid, fasting blood glucose [FBG], glycosylated hemoglobin levels) were also recorded. The severity of PD was assessed by Hoehn-Yahr (H-Y) staging and the Movement Disorder Society-Unified Parkinson's Disease Rating Scale part III (MDS-UPDRS III) total score. The subscores for tremor (items 3.15, 3.16, 3.17 and 3.18), rigidity (items 3.3), bradykinesia (items $3.4,3.5,3.6,3.7,3.8,3.9$ and 3.14) and gait/postural instability (items $3.10,3.11$ and 3.12) were obtained from the MDS-UPDRS III. Cognition was assessed by the Montreal Cognitive Assessment (MoCA). Mood was assessed by the Hamilton Anxiety Rating Scale (HAMA) and Hamilton Depression Rating Scale (HAMD). Motor assessment was performed in an off-medication state; however, cognition and mood assessments were performed in an on/off-medication state. A score of at least one point on the 19-item Wearing-off Questionnaire (WOQ-19) was defined as the possible presence of the WO. The WOQ-19, which covers a wide range of 
symptoms and emphasize the importance of certain easier neglected symptoms, such as anxiety, numbness and cold and hot feelings, has a good accuracy. The levodopa equivalent daily dosage (LEED) was calculated as levodopa dose +levodopa dose $\times 1 / 3$ if on entacapone + piribedil $(\mathrm{mg})+$ pramipexole $(\mathrm{mg}) \times 100+$ selegiline $(\mathrm{mg}) \times 10+$ amantadine $(\mathrm{mg})+$ controlled-release levodopa $(\mathrm{mg}) \times 0.75$.

\section{MRI Assessment}

MRI data were acquired from four 3-T MRI and one 1.5-T MRI devices in Beijing Tiantan Hospital with T1weighted imaging, T2-weighted imaging, fluid-attenuated inversion recovery imaging (FLAIR), and susceptibilityweighted imaging (SWI) sequences. The MRI data were collected and evaluated in Beijing Tiantan Hospital. All image readers were professionally trained and blinded to each other and to the patients' demographic information and clinical data. The same images were interpreted by two readers, and a final review by a senior radiologist to ensure the accuracy and consistency of the imaging findings.

Lacunae were defined as round or ovoid cavities with diameters ranging from $3 \mathrm{~mm}$ to $15 \mathrm{~mm}$ and were characterized by a hypointense lesion on FLAIR images, according to the corresponding hyper- and hypo- intensities on T2- and T1-weighted images, respectively. ${ }^{19}$ The presence and number of lacunae were examined in the subcortical white matter (including the internal capsule, corona radiata, and corpus callosum), basal ganglia (including the caudate nuclei, and lenticular nuclei), thalamus and infratentorial area. In addition, to adjust for their potential influence on the WO, other biomarkers of CSVD, such as WMH, enlarged perivascular space (PVS) and microbleeds (MBs), were also assessed. WMH was assessed by the Fazekas scale ${ }^{20}$ and divided into two groups, periventricular hyperintensities (PVH) and deep white matter hyperintensities (DWMH), both of which were graded on a scale of $0-3$. The total score of the Fazekas scale was the sum of the PVH score and the DWMH score. PVSs in the basal ganglia (BG-PVSs) and in the centrum semiovale (CS-PVSs) were rated separately according to the semiquantitative rating scale developed by the Edinburg group. ${ }^{21}$ MBs were assessed according to the Microbleed Anatomical Rating Scale (MARS). ${ }^{22}$

\section{Statistical Analysis}

All statistical analyses were performed in SPSS 24.0. The Kolmogorov-Smirnov test was used to check the distribution of the data. Continuous variables were expressed as the means and standard deviations (SD) or interquartile range (IQR), and categorical variables were reported as numbers and percentages. The student's $t$-test and the Mann-Whitney $U$-test were used for comparison of normally and abnormally distributed data, respectively. The Chi-square test was used to evaluate the differences in categorical variables. A binary logistic regression model was conducted to explore the potential influence of lacunae on the $\mathrm{WO}$ in $\mathrm{PD} . \mathrm{P}<0.05$ is considered statistically significant.

\section{Results}

A total of 315 PD patients were enrolled in the current study, and 191 (61.63\%) PD patients reported experiencing the WO. The demographic and clinical characteristics, cerebrovascular disease-related risk factors and CSVD-related MRI features of the patients are listed in Table 1.

\section{Differences Between PD Patients with} and without the WO

The differences in the demographic and clinical characteristics, cerebrovascular disease-related risk factors and CSVD-related MRI features between patients with and without the WO are presented in Table 1. Patients with the WO showed a significantly earlier onset age, longer disease duration, greater disease stage, higher motor and mood scores and higher LEED $(\mathrm{P}<0.01)$. The gait/postural instability subscores were higher in patients with the WO $(\mathrm{P}<0.05)$; however, there were no differences in the tremor, rigidity or bradykinesia subscores between patients with and without the WO $(\mathrm{P}>0.05)$. Patients with the WO had significantly higher serum HCY levels and lower serum uric acid levels than patients without the WO $(\mathrm{P}<0.05)$. A significant difference in the number of lacunae was found between patients with and without the WO $(\mathrm{P}<0.05$, Figure 1). Other CSVD imaging biomarkers were not significantly different between the two groups $(\mathrm{P}>0.05$, Figure 1).

\section{Association Between Lacune and WO in PD Patients}

Binary logistic regression showed that the number of lacunae were significantly associated with the WO in PD patients according to the model 1 (adjusted for age at onset, disease duration, $\mathrm{H}-\mathrm{Y}$ staging, MDS-UPDRS III total score and LEED) $(\mathrm{P}=0.037$, OR 1.156, 95\% CI 1.009, 1.325) and model 2 (model 1+adjusted for WMH, PVS, MBs) 
Table I Differences Between PD Patients with/Without the WO

\begin{tabular}{|c|c|c|c|c|}
\hline & Total $(n=3 \mid 5)$ & With WO $(n=191)$ & Without WO $(n=124)$ & $P$ value \\
\hline \multicolumn{5}{|l|}{ Demographic features } \\
\hline Age at admission, years, median (IQR) & $64.00(58.00,69.00)$ & $64.00(58.00,69.00)$ & $65.00(58.00,69.75)$ & 0.312 \\
\hline Sex, $n(F / M)$ & $131 / 184$ & $85 / 106$ & $46 / 78$ & 0.193 \\
\hline BMI, median (IQR) & $24.22(21.99,26.45)$ & $24.22(21.99,26.37)$ & $24.05(21.80,26.54)$ & 0.537 \\
\hline \multicolumn{5}{|l|}{ Medical history, n (\%) } \\
\hline Hypertension & $96(30.48 \%)$ & $53(27.75 \%)$ & $43(34.68 \%)$ & 0.192 \\
\hline Diabetes mellitus & $38(12.06 \%)$ & $23(12.04 \%)$ & $15(12.10 \%)$ & 0.988 \\
\hline Coronary heart disease & $32(10.16 \%)$ & $23(12.04 \%)$ & $9(7.26 \%)$ & 0.170 \\
\hline Current or previous smoking & $93(29.52 \%)$ & $50(26.18 \%)$ & $43(34.68 \%)$ & 0.106 \\
\hline Current or previous drinking & 77 (24.44\%) & $43(22.51 \%)$ & $34(27.42 \%)$ & 0.322 \\
\hline \multicolumn{5}{|l|}{ Blood test at admission } \\
\hline TG, mmol/L, median (IQR) & $1.03(0.74,1.47)$ & $1.00(0.74,1.44)$ & $1.05(0.72,1.53)$ & 0.818 \\
\hline $\mathrm{TC}, \mathrm{mmol} / \mathrm{L}$, mean $\pm \mathrm{SD}$ & $4.06 \pm 0.79$ & $4.06 \pm 0.80$ & $4.06 \pm 0.78$ & 0.967 \\
\hline $\mathrm{LDL}, \mathrm{mmol} / \mathrm{L}$, mean $\pm \mathrm{SD}$ & $2.4 I \pm 0.69$ & $2.39 \pm 0.70$ & $2.45 \pm 0.67$ & 0.469 \\
\hline HDL, mmol/L, median (IQR) & $1.18(1.08,1.32)$ & $1.22(1.04 .1 .40)$ & $1.16(0.98,1.34)$ & 0.080 \\
\hline HCY, $\mu \mathrm{mol} / \mathrm{L}$, median (IQR) & $14.88(12.32,18.41)$ & $15.35(12.58,19.45)$ & $14.73(11.67,17.35)$ & $0.035^{*}$ \\
\hline Uric acid, $\mu \mathrm{mol} / \mathrm{L}$, median (IQR) & $282.20(232.8,323.4)$ & $270.30(223.80,317.50)$ & $293.35(250.43,344.88)$ & $0.002 * *$ \\
\hline FBG, mmol/L, median (IQR) & $4.53(4.22,4.99)$ & $4.55(4.21,5.06)$ & $4.52(4.29,4.91)$ & 0.778 \\
\hline Glycosylated hemoglobin, \%, median (IQR) & $5.7(5.5,6.0)$ & $5.70(5.40,6.00)$ & $5.70(5.50,6.00)$ & 0.700 \\
\hline \multicolumn{5}{|l|}{ Clinical features } \\
\hline Age at onset, years, median (IQR) & $57.00(50.00,63.00)$ & $55.00(50.00,62.00)$ & $59.00(52.00,65.00)$ & $0.001 * *$ \\
\hline Disease duration, years, median (IQR) & $6.00(4.00,10.00)$ & $8.00(5.00,10.00)$ & $4.00(3.00,7.00)$ & $<0.001 * *$ \\
\hline $\mathrm{H}-\mathrm{Y}$ staging, median (IQR) & $3.00(2.00,4.00)$ & $3.00(2.50,4.00)$ & $3.00(2.00,3.00)$ & $<0.001 * *$ \\
\hline MDS-UPDRS III total score, median (IQR) & $39.00(29.00,52.00)$ & $43.00(32.00,54.00)$ & $36.00(24.00,45.75)$ & $<0.001 * *$ \\
\hline Tremor subscore, median (IQR) & $6.00(2.00,10.00)$ & $6.00(2.00,11.00)$ & $5.00(1.00,9.00)$ & 0.200 \\
\hline Rigidity subscore, median (IQR) & $8.00(4.00,10.50)$ & $8.00(4.00,11.00)$ & $7.00(4.00,10.00)$ & 0.147 \\
\hline Bradykinesia subscore, mean $\pm S D$ & $24.43 \pm 12.89$ & $25.61 \pm 13.19$ & $22.31 \pm 12.12$ & 0.056 \\
\hline Gait and postural subscore, median (IQR) & $4.00(2.00,6.00)$ & $4.00(2.00,7.00)$ & $3.00(1.00,5.00)$ & $0.007 * *$ \\
\hline LEED, mg/day, median (IQR) & $500.00(300.00,700.00)$ & $550.00(375.00,750.00)$ & $400.00(250.00,600.00)$ & $0.001 * *$ \\
\hline MoCA score, median (IQR) & $22.00(17.00,26.00)$ & $22.00(17.00,25.00)$ & $23.00(18.75,26.00)$ & 0.143 \\
\hline HAMA score, median (IQR) & $13.00(8.00,19.00)$ & $14.00(9.00,20.00)$ & $10.00(6.00,17.00)$ & $0.003 * *$ \\
\hline HAMD score, median (IQR) & $12.00(6.00,18.00)$ & $14.00(7.00,20.00)$ & $10.00(5.00,16.00)$ & $0.005^{* *}$ \\
\hline
\end{tabular}

Notes: ${ }^{*} p<0.05, * * p<0.01$.

Abbreviations: WO, wearing-off phenomenon; IQR, interquartile range; SD, standard deviations; BMI, body mass index; TG, triglyceride; TC, total cholesterol; LDL, lowdensity lipoprotein; HDL, high-density lipoprotein; HCY, homocysteine; FBG, fasting blood glucose; H-Y staging, Hoehn-Yahr staging; MDS-UPDRS III, Movement Disorder Society-Unified Parkinson's Disease Rating Scale part III; LEED, levodopa equivalent daily dosage; MMSE, Mini-Mental State Examination; MoCA, Montreal Cognitive Assessment; HAMA, Hamilton Anxiety Rating Scale; HAMD, Hamilton Depression Rating Scale.

$(\mathrm{P}=0.046$, OR 1.172, 95\% CI 1.003, 1.369). After further adjustment for other potential confounders (model 3, model $2+$ adjusted for age at admission, sex, BMI, risk factors for cerebrovascular disease and MRI machines), the association remained significant $(\mathrm{P}=0.043$, OR $1.195,95 \%$ CI 1.005 , 1.421) (Table 2).

\section{Association of the Location of the Lacunae with the WO in PD Patients}

As shown in Table 3, we explored the association between the location of the lacunae and the WO in PD patients. Lacunae in subcortical areas $(\mathrm{P}=0.004, \mathrm{OR} 0.498,95 \%$ CI $0.308,0.803)$ and basal ganglia $(\mathrm{P}=0.046$, OR 1.616, 95\% CI 1.009, 2.587), especially in the caudate nuclei $(\mathrm{P}=0.023$, OR $1.104,95 \% \mathrm{CI}$ $0.185,0.881)$, were significantly associated with the WO in PD patients (Table 3).

\section{Discussion}

The present study investigated the association between lacunae and the WO in PD patients from a new perspective involving the cross-field of cerebrovascular disease and neurodegenerative disease. The results showed that lacunae were significantly associated with the WO in PD patients independent of age, disease duration, 

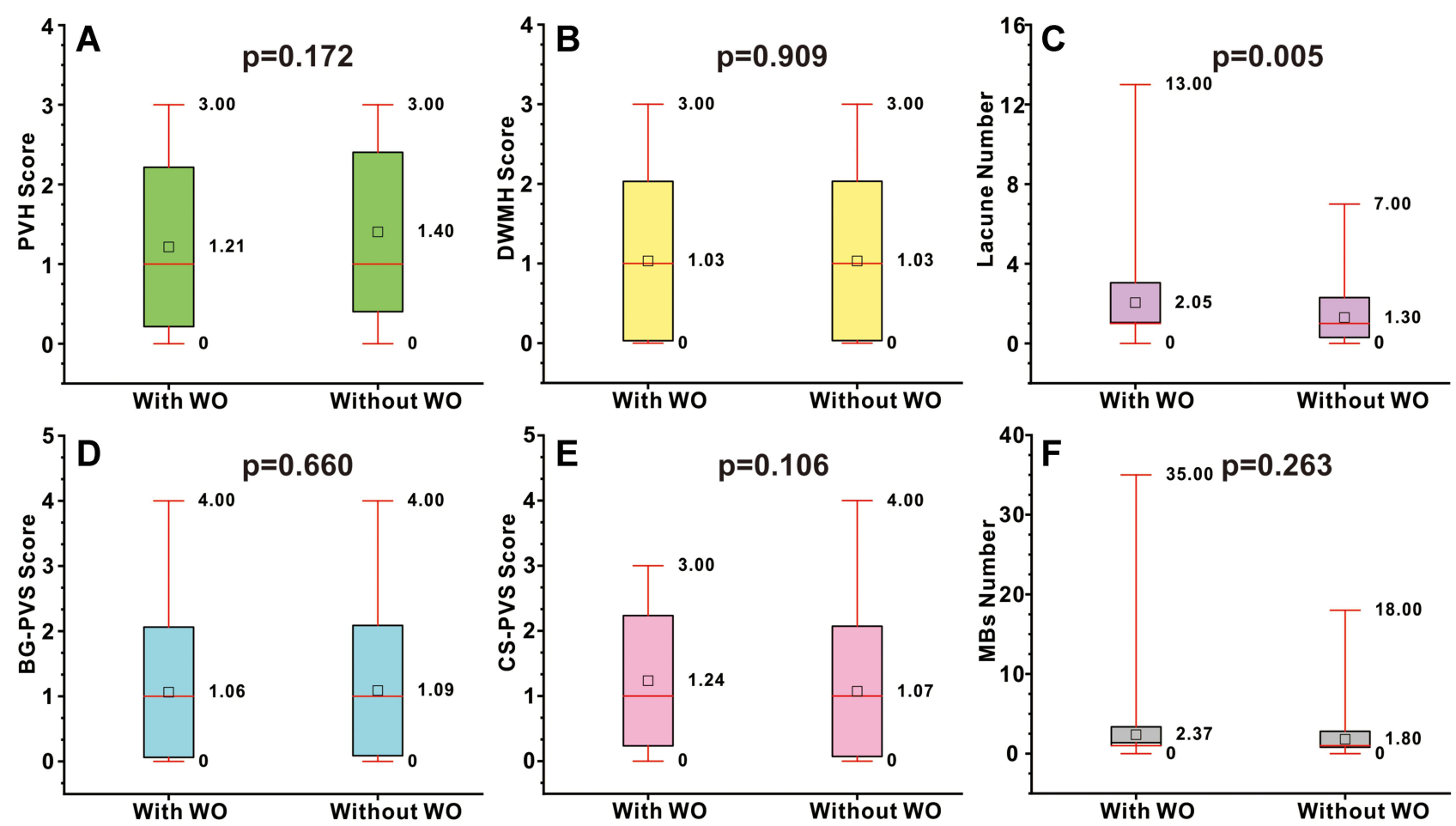

Figure I CSVD related MRI features between PD patients with/without the WO. Boxplot (A-F) showed the CSVD related MRI features between PD patients with/without the WO, including PVH, DWMH, lacunae number, BG-PVS, CS-PVS and MBs.

Abbreviations: CSVD, cerebral small vessel disease; PVH, periventricular hyperintensities; DWMH, deep white matter hyperintensities; BG-PVS, enlarged perivascular space in the basal ganglia; CS-PVS, enlarged perivascular space in the centrum semiovale; MBs, microbleeds.

H-Y staging, MDS-UPDRS III score, LEED, and serum HCY and uric acid levels. Furthermore, we found that lacunae in subcortical areas and the caudate nuclei were associated with the WO in PD patients.

As the name implies, the wearing-off phenomenon, a common motor fluctuation in PD patients as the disease progresses, is characterized by the development of a loss of response to the medication dose before taking the next dose. A pharmacokinetic study indicated that patients

Table 2 Association Between Lacunae and the WO in PD Patients

\begin{tabular}{|l|l|l|l|}
\hline & $\mathbf{R}^{\mathbf{2}}$ & OR $\mathbf{( 9 5 \%} \mathbf{~ C I})$ & P value \\
\hline Model I & 0.155 & $\mathrm{I} .156(\mathrm{I} .009, \mathrm{I} .325)$ & $0.037^{*}$ \\
Model 2 & 0.173 & $\mathrm{I} .172(\mathrm{I} .003, \mathrm{I} .369)$ & $0.046^{*}$ \\
Model 3 & 0.257 & $\mathrm{I} .195(\mathrm{I} .005, \mathrm{I} .42 \mathrm{I})$ & $0.043^{*}$ \\
\hline
\end{tabular}

Notes: All variables were entered into the logistic models simultaneously. Model I adjusted for age at onset, disease duration, $\mathrm{H}-\mathrm{Y}$ staging, MDS-UPDRS III total score and LEED. Model 2= model I+adjusted WMH, PVS, MBs. Model 3= model 2+ adjusted for age at admission, sex, MoCA, HAMA, HAMD, BMI, risk factors for cerebrovascular disease and MRI machines. ${ }^{*} \mathrm{p}<0.05$.

Abbreviations: OR, odds ratio; $\mathrm{Cl}$, confidence interval; $\mathrm{H}-\mathrm{Y}$ staging, Hoehn-Yahr staging; MDS-UPDRS III, Movement Disorder Society-Unified Parkinson's Disease Rating Scale part III; LEED, levodopa equivalent daily dosage; $\mathrm{WMH}$, white matter hyperintensity; PVS, enlarged perivascular space; MBs, microbleeds; BMI, body mass index. usually showed a good response after taking a single dose of levodopa for 60 minutes, but the parkinsonism symptoms re-emerged or were exacerbated before their next scheduled dose. ${ }^{23}$ The most common explanation is that the benefit of the last dose "wears-off". The exact mechanism of the WO has not been well clarified, and may be multifactorial. It is generally accepted that the degeneration of presynaptic dopaminergic neurons, results in a lack of storage for levodopa and a resultant inability to buffer variations in levodopa availability. ${ }^{24}$ Postsynaptically, alterations in receptor responsiveness and changes in the pharmacodynamic response may also be responsible mechanisms. ${ }^{25}$ In addition to the central mechanism, peripheral factors such as high-protein $\operatorname{diets}^{26}$ and gastrointestinal dysfunction ${ }^{27}$ could also influence the absorption of levodopa and induce WO.

In our study, the prevalence of the WO was $61.63 \%$, higher than that in a study in mainland China. ${ }^{28}$ The possible reason for this was that in the earlier study, the authors screened the WO in their study with the WOQ-9, which has a lower specificity in identifying WO. ${ }^{29}$

In contrast to previous studies on the risk factors for the WO, our study found that lacunae were a predictable 
Table 3 The Association of the Location of the Lacunae with the WO in PD Patients

\begin{tabular}{|l|l|l|l|l|}
\hline & $\mathbf{R}^{\mathbf{2}}$ & $\mathbf{O R}$ & $\mathbf{9 5 \%} \mathbf{~ I ~}$ & P value \\
\hline Lacunae in subcortical areas & 0.036 & 0.498 & $0.308,0.803$ & $0.004^{* *}$ \\
Lacunae in basal ganglia & 0.017 & 1.616 & $1.009,2.587$ & $0.046^{*}$ \\
Lacunae in lenticular nucleus & 0.001 & 0.901 & $0.570,1.422$ & 0.653 \\
Lacunae in caudate nucleus & 0.025 & 1.104 & $0.185,0.881$ & $0.023^{*}$ \\
Lacunae in thalamus & 0.013 & 0.567 & $0.291,1.104$ & 0.095 \\
Lacunae in subtentorial areas & 0.012 & 0.553 & $0.272,1.123$ & 0.101 \\
\hline
\end{tabular}

Notes: All variables were entered into the logistic models simultaneously. ${ }^{*} p<0.05,{ }^{*} *_{p}<0.01$.

Abbreviations: $\mathrm{OR}$, odds ratio; $\mathrm{Cl}$, confidence interval.

factor, especially those located in the caudate nuclei. The imaging definition of a lacune is a round or ovoid, subcortical, fluid-filled (similar signal as CSF) cavity, measuring between $3 \mathrm{~mm}$ and approximately $15 \mathrm{~mm}$ in diameter, consistent with a previous acute small deep brain infarct or hemorrhage in the territory of one of the perforating arterioles. $^{19}$ Blood-brain barrier leakage and endothelial dysfunction seem to be pivotal factors contributing to the pathogenesis of lacunae or CSVD. ${ }^{10}$

We hypothesized that the number and location of lacunae may be correlated with the presynaptic and postsynaptic mechanisms of the WO. First, a lacunar lesion located in the basal ganglia, especially in the caudate nuclei, the main brain area involved in PD, may worsen the degeneration of presynaptic dopaminergic neurons and further affect the release of levodopa. Additionally, it may alter the binding capacity of dopamine receptors in the postsynaptic membrane of dopamine neurons. These factors would affect the continuous and stable release of the levodopa, and resulting in the emergence of the WO. Second, a lacunar lesion may likely disrupt the striatothalamo-cortical pathway, a well-established functional pathway in PD. ${ }^{30,31}$ This pathway is related to the motor symptoms in $\mathrm{PD}$, and potentially to the WO as the disease progresses and the pathway is disrupted. In the lesion network analysis by Joutsa et al, lesional locations associated with parkinsonism were functionally connected to a common network similar to the network affected in idiopathic PD. ${ }^{32}$ Future studies with functional imaging or molecular imaging are needed to test this hypothesis. Furthermore, the common mechanism of neurovascular unit dysfunction of the lacunae and PD may also contribute to the association between the lacunae and the WO in PD patients. $^{33}$

We found that patients with the WO had an earlier onset age, longer disease duration, greater disease stage, higher motor and mood scores and higher LEED, which were consistent with previous study. ${ }^{4,5}$ We also found that the gait/postural instability subscores were higher in patients with the WO, which may mean that a higher gait/postural instability subscores or postural instability/ gait-dominant motor phenotype could be a risk factor for WO. In addition, our study showed that patients with the WO had higher HCY levels and lower uric acid levels. The association between HCY or uric acid and the WO has not been very clarified. Only one study showed that serum uric acid levels were inversely associated with the occurrence of the WO in males with PD, ${ }^{34}$ consistent with the findings in our study. The authors supposed that uric acid has a neuroprotective effect on presynaptic terminals and in preventing the WO in PD. More related studies should be conducted to explore this relationship in the future.

Our study has several implications. First, the comorbidity of the lacunae in PD requires greater attention and intervention, as a silent lacune is not actually silent. Second, the WO in PD may be multifactorial, and related to additional factors beyond demographic and clinical features. Third, lacunae may share a common mechanism with PD, rather than causing motor symptoms and worsening of the WO. Fourth, promoting neurovascular health may prevent the progression of the WO in PD.

The study has several strengths. First, to the best of our knowledge, this is the first study from the perspective of CSVD to investigate predictive factors of the WO in PD. Second, a relatively large sample size was used in our study. Third, we also investigated the correlation of the location of lacunar lesions with the WO. Fourth, we used several regression models to understand the effect of lacunae on the WO in PD.

Several limitations of the present retrospective study also need to be acknowledged. First, the retrospective study design may be affected by recall and selection bias, and the fact that the admitted PD patients had may 
affect the generalizability of the results. However, physicians can directly assess the fluctuation of symptoms and minimize the chance of underdiagnosing the WO in admitted hospital PD patients. Future longitudinal studies and larger samples are needed. Second, WO-related symptoms vary from motor symptoms to nonmotor symptoms. PD patients with early-stage WO may be unaware of the subtle motor fluctuations related to PD treatment and thus may not bring them to the attention of their physicians, thus leading to an underdiagnosis of the WO. Although we used the WOQ-19, a suggested screening scale for MDS, ${ }^{29}$ to assess the presence of the WO, this questionnaire was unable to assess the severity of the WO. Therefore, quantitative assessments, such as the MDS-UPDRS, should be performed during on- and off-medication states to identify symptom fluctuations. Third, the diagnosis of PD in this study was based on clinical diagnostic criteria rather than pathology, which may affect the diagnostic accuracy for the disease.

\section{Conclusion}

In conclusion, lacunae may be independent contributors to the WO in PD patients, possibly by affecting the presynaptic and postsynaptic mechanisms of the phenomenon. Promoting neurovascular health may prevent the progression of the WO in PD.

\section{Data Sharing Statement}

The datasets used and/or analyzed in this study are available from the corresponding authors upon reasonable request.

\section{Ethics Approval and Informed Consent}

This study was approved by the Ethics Committee of the Beijing Tiantan Hospital and was performed in accordance with the Declaration of Helsinki. Informed consent was obtained either from the participants or their closest relatives.

\section{Acknowledgments}

The authors thank all the participants who were enrolled in this study.

\section{Funding}

The study was supported by grants from the National Key Technology Research and Development Program of China (2018YFC2002300, 2018YFC2002302, 2020YFC2004102), and the National Natural Science Foundation of China (NSFC:81972144, 31872785, 81972148).

\section{Disclosure}

The authors report no conflicts of interest.

\section{References}

1. Armstrong M, Okun M. Diagnosis and treatment of Parkinson disease: a review. JAMA. 2020;323(6):548-560. doi:10.1001/ jama.2019.22360

2. Stocchi F, Antonini A, Barone P, et al. Early DEtection of wEaring off in Parkinson disease: the DEEP study. Parkinsonism Relat Disord. 2014;20(2):204-211. doi:10.1016/j.parkreldis.2013.10.027

3. Ahlskog J, Muenter M. Frequency of levodopa-related dyskinesias and motor fluctuations as estimated from the cumulative literature. Mov Disord. 2001;16(3):1683. doi:10.1212/WNL.43.9.1683

4. Warren Olanow C, Kieburtz K, Rascol O, et al. Factors predictive of the development of levodopa-induced dyskinesia and wearing-off in Parkinson's disease. Mov Disord. 2013;28(8):1064-1071. doi: $10.1002 / \mathrm{mds} .25364$

5. Raja K, Ramrakhia S, Dev K, et al. The risk factors for the wearingoff phenomenon in Parkinson's disease. Cureus. 2020;12(9):e10729. doi:10.7759/cureus.10729

6. Pozorski V, Oh JM, Okonkwo O, et al. Cross-sectional and longitudinal associations between total and regional white matter hyperintensity volume and cognitive and motor function in Parkinson's disease. Neuroimage Clin. 2019;23:101870. doi:10.1016/j.nicl.2019.101870

7. Chen H, Wan H, Zhang M, et al. Cerebral small vessel disease may worsen motor function, cognition, and mood in Parkinson's disease. Parkinsonism Relat Disord. 2021;83:86-92. doi:10.1016/j. parkreldis.2020.12.025

8. Wan $\mathrm{Y}, \mathrm{Hu} \mathrm{W}$, Gan J, et al. Exploring the association between cerebral small-vessel diseases and motor symptoms in Parkinson's disease. Brain Behav. 2019;9(4):e01219. doi:10.1002/brb3.1219

9. Toda K, Iijima M, Kitagawa K. Periventricular white matter lesions influence gait functions in Parkinson's disease. Eur Neurol. 2019;81 (3-4):120-127. doi:10.1159/000499908

10. Cuadrado-Godia E, Dwivedi P, Sharma S, et al. Cerebral Small vessel disease: a review focusing on pathophysiology, biomarkers, and machine learning strategies. $J$ Stroke. 2018;20(3):302-320. doi:10.5853/jos.2017.02922

11. Allali G, Blumen H, Devanne H, Pirondini E, Delval A, Van De Ville D. Brain imaging of locomotion in neurological conditions. Neurophysiol Clin. 2018;48(6):337-359. doi:10.1016/j. neucli.2018.10.004

12. Levit A, Hachinski V, Whitehead S. Neurovascular unit dysregulation, white matter disease, and executive dysfunction: the shared triad of vascular cognitive impairment and Alzheimer disease. GeroScience. 2020;42(2):445-465. doi:10.1007/s11357-020-00164-6

13. Schwartz R, Halliday G, Cordato D, Kril J. Small-vessel disease in patients with Parkinson's disease: a clinicopathological study. Mov Disord. 2012;27(12):1506-1512. doi:10.1002/mds.25112

14. Marras C, Canning C, Goldman S. Environment, lifestyle, and Parkinson's disease: implications for prevention in the next decade. Mov Disord. 2019;34(6):801-811. doi:10.1002/mds.27720

15. Veselý B, Antonini A, Rektor I. The contribution of white matter lesions to Parkinson's disease motor and gait symptoms: a critical review of the literature. J Neural Transm. 2016;123(3):241-250. doi:10.1007/s00702-015-1470-9

16. Veselý B, Rektor I. The contribution of white matter lesions (WML) to Parkinson's disease cognitive impairment symptoms: a critical review of the literature. Parkinsonism Relat Disord. 2016;S166-70. doi:10.1016/j.parkreldis.2015.09.019 
17. Chen H, Zhang M, Liu G, et al. Effect of small vessel disease burden and lacunes on gait/posture impairment in Parkinson's disease. Neurol Sci. 2020;41(12):3617-3624. doi:10.1007/s10072-02004452-z

18. Postuma R, Berg D, Stern M, et al. MDS clinical diagnostic criteria for Parkinson's disease. Mov Disord. 2015;30(12):1591-1601. doi:10.1002/mds. 26424

19. Wardlaw JM, Smith EE, Biessels GJ, et al. Neuroimaging standards for research into small vessel disease and its contribution to ageing and neurodegeneration. Lancet Neurol. 2013;12(8):822-838. doi:10.1016/s1474-4422(13)70124-8

20. Fazekas F, Kleinert R, Offenbacher H, et al. Pathologic correlates of incidental MRI white matter signal hyperintensities. Neurology. 1993;43(9):1683-1689. doi:10.1212/wnl.43.9.1683

21. Doubal F, MacLullich A, Ferguson K, Dennis M, Wardlaw J. Enlarged perivascular spaces on MRI are a feature of cerebral small vessel disease. Stroke. 2010;41(3):450-454. doi:10.1161/ strokeaha.109.564914

22. Gregoire S, Chaudhary U, Brown M, et al. The Microbleed Anatomical Rating Scale (MARS): reliability of a tool to map brain microbleeds. Neurology. 2009;73(21):1759-1766. doi:10.1212/ WNL.0b013e3181c34a7d

23. Fabbrini G, Juncos J, Mouradian M, Serrati C, Chase T. Levodopa pharmacokinetic mechanisms and motor fluctuations in Parkinson's disease. Ann Neurol. 1987;21(4):370-376. doi:10.1002/ana.410 210409

24. Fabbrini G, Mouradian M, Juncos J, Schlegel J, Mohr E, Chase T. Motor fluctuations in Parkinson's disease: central pathophysiological mechanisms, part I. Ann Neurol. 1988;24(3):366-371. doi:10.1002/ ana.410240303

25. Mouradian M, Juncos J, Fabbrini G, Schlegel J, Bartko J, Chase T. Motor fluctuations in Parkinson's disease: central pathophysiological mechanisms, part II. Ann Neurol. 1988;24(3):372-378. doi:10.1002/ ana.410240304
26. Wang L, Xiong N, Huang J, et al. Protein-restricted diets for ameliorating motor fluctuations in Parkinson's disease. Front Aging Neurosci. 2017;9:206. doi:10.3389/fnagi.2017.00206

27. Doi H, Sakakibara R, Kishi M, Tsuyuzaki Y, Tateno F, Hirai S. [Gastrointestinal dysfunction has important implications for plasma L-dopa concentrations in Parkinson's disease]. Rinsho Shinkeigaku. 2013;53(11):1382-1385. Japanese. doi:10.5692/ clinicalneurol.53.1382

28. Chen W, Xiao Q, Shao M, et al. Prevalence of wearing-off and dyskinesia among the patients with Parkinson's disease on levodopa therapy: a multi-center registry survey in mainland China. Transl Neurodegener. 2014;3(1):26. doi:10.1186/2047-9158-3-26

29. Antonini A, Martinez-Martin P, Chaudhuri RK, et al. Wearing-off scales in Parkinson's disease: critique and recommendations. Mov Disord. 2011;26(12):2169-2175. doi:10.1002/mds.23875

30. Chen H, Sha Z, Ma H, He Y, Feng T. Effective network of deep brain stimulation of subthalamic nucleus with bimodal positron emission tomography/functional magnetic resonance imaging in Parkinson's disease. CNS Neurosci Ther. 2018;24(2):135-143. doi:10.1111/ cns. 12783

31. Albin R, Young A, Penney J. The functional anatomy of disorders of the basal ganglia. Trends Neurosci. 1995;18(2):63-64. doi:10.1016/ 0166-2236(95)93872-u

32. Joutsa J, Horn A, Hsu J, Fox M. Localizing parkinsonism based on focal brain lesions. Brain. 2018;141(8):2445-2456. doi:10.1093/ brain/awy 161

33. Zlokovic BV. The blood-brain barrier in health and chronic neurodegenerative disorders. Neuron. 2008;57(2):178-201. doi:10.1016/j. neuron.2008.01.003

34. Fukae J, Ishikawa K, Hatano T, et al. Serum uric acid concentration is linked to wearing-off fluctuation in Japanese Parkinson's disease patients. J Parkinsons Dis. 2014;4(3):499-505. doi:10.3233/JPD140353
Neuropsychiatric Disease and Treatment

\section{Publish your work in this journal}

Neuropsychiatric Disease and Treatment is an international, peerreviewed journal of clinical therapeutics and pharmacology focusing on concise rapid reporting of clinical or pre-clinical studies on a range of neuropsychiatric and neurological disorders. This journal is indexed on PubMed Central, the 'PsycINFO' database and CAS, and

\section{Dovepress}

is the official journal of The International Neuropsychiatric Association (INA). The manuscript management system is completely online and includes a very quick and fair peer-review system which is all easy to use. Visit http://www.dovepress.com/testimonials.php to read real quotes from published authors. 